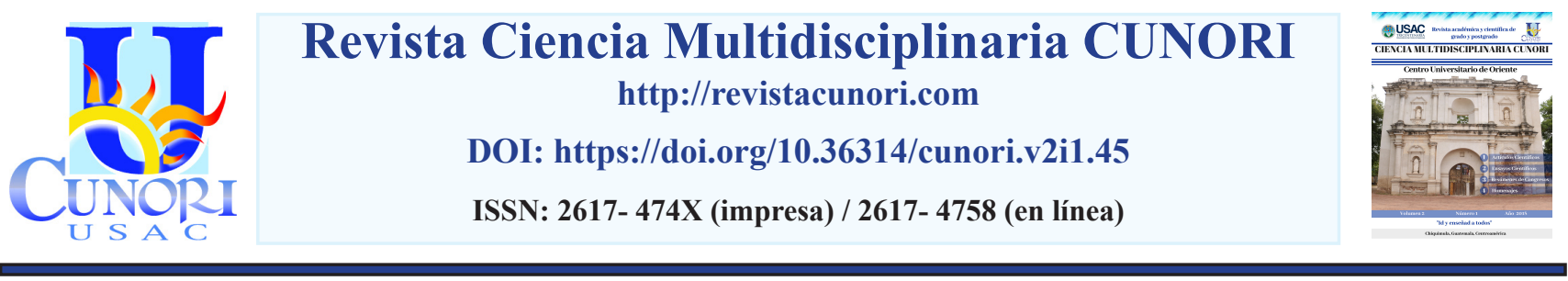

Como citar el artículo

García K., Osorio C., Retana R. Mazariegos E.,Arriola C., et al. (2018). Disfunción tiroidea en pacientes infectados por VIH con terapia antirretroviraldegranactividad.RevistaCienciaMultidisciplinariaCUNORI,2(1), 1-6.https://doi.org/10.36314/cunori.v2i1.45

\title{
Disfunción tiroidea en pacientes infectados por VIH con terapia antirretroviral de gran actividad
}

\section{Thyroid dysfunction in HIV infected patients with highly active antiretroviral therapy}

\author{
Kristha García*, Carlos Osorio, Claudia Mazariegos, Carlos Arriola, Ronaldo Retana \& Edvin Mazariegos \\ Centro Universitario de Oriente (CUNORI), Universidad de San Carlos de Guatemala \\ Recibido: 17 de junio de 2018 / Aceptado: 08 de agosto de 2018 \\ Disponible en internet el 17 de agosto de 2018
}

*Autor para correspondencia.

Correo electrónico: kristha_hello@hotmail.com

\section{Resumen}

$\mathrm{L}$ a disfunción tiroidea puede ser común en la infección por VIH y también en casos de uso de terapia antirretroviral de gran actividad. El estudio es analítico transversal, el cual determinó la relación de disfunción tiroidea en pacientes infectados por VIH con terapia antirretroviral de gran actividad asociándolo con la duración de la terapia, recuento de CD4 y carga viral en la Clínica de Atención Integral de Zacapa, Guatemala. Con un muestreo probabilístico aleatorio de 218 pacientes estratificándolos en subgrupos en relación a los años de uso de terapia antirretroviral de gran actividad, se les realizó TSH y T4 libre, asociándolo con tiempo de la terapia antirretroviral, carga viral y CD4. Se determinó la asociación entre la duración del uso de la Terapia Antirretroviral de Gran Actividad-TARGA, y la prevalencia de disfunción tiroidea $(\mathrm{p}<0.001)$. La profilaxis administrada para prevenir infecciones oportunistas se asoció al hipotiroidismo subclínico $(\mathrm{p}=0.004 ; \mathrm{RP}=3.81)$. Los pacientes con hipotiroidismo subclínico tuvieron una mayor intensidad de síntomas como insomnio, piel seca, entre otros. Se logró comprobar que la presencia de hipotiroidismo subclínico se relaciona con el tiempo de uso de antirretrovirales, y con el uso de profilaxis para infecciones oportunistas, lo cual se puede deber al compromiso inmunológico que presenta este tipo de pacientes, pudiendo tener efectos sobre el sistema endocrino, o una complicación endocrinológica propia de la terapia .

Palabras clave: VIH, disfunción tiroidea, TARGA (terapia antirretroviral de gran actividad)

Abstract

$\mathrm{T}$ hyroid dysfunction may be common in HIV infection and also in cases of highly active antiretroviral therapy. The study is cross-sectional analysis, which determined the relationship of thyroid dysfunction in HIV-infected patients with highly active antiretroviral therapy associated with the duration of therapy, CD4 count and viral load in the Comprehensive Care Clinic of Zacapa, Guatemala. With randomized probabilistic sampling of 218 patients stratifying them into subgroups in relation to the years of use of highly active antiretroviral therapy, TSH and free T4 were performed, associating it with time of antiretroviral therapy, viral load and CD4. The association between the duration of the use of Antiretroviral Therapy of Great Activity-TRARGA, and the prevalence of thyroid dysfunction $(\mathrm{p}<0.001)$ was determined.Prophylaxis administered to prevent opportunistic infections was associated with subclinical hypothyroidism $(\mathrm{p}=0.004, \mathrm{RP}=3.81)$. Patients with subclinical hypothyroidism had a higher intensity of symptoms such as insomnia, dry skin, among others. It was verified that the presence of subclinical hypothyroidism is related to the time of antiretroviral use, and to the use of prophylaxis for opportunistic infections, which may be due to the immunological compromise that this type of patients presents, and may have effects on the system. endocrine, or an endocrine complication typical of therapy. 
Keywords: HIV, thyroid dysfunction, HAART (highly active antiretroviral therapy)

\section{Introducción}

La infección por VIH por su impacto en el sistema inmune podría asociarse a manifestaciones endocrinológicas, las que podrían afectar la evolución de la enfermedad manifestándose como disfunción tiroidea; ya que, el sistema inmune está modulado por efecto hormonal, así como también la respuesta inmune es capaz de afectar el sistema endocrino. Algunos estudios han sugerido que la prevalencia de enfermedad tiroidea en pacientes con VIH es del 17\%. (Báez, et al. 2016). Por lo cual, se buscó determinar la relación de disfunción tiroidea en pacientes infectados por VIH con TARGA asociándolo con la duración de la terapia, recuento de células CD4 y carga viral, ya que en la actualidad las tendencias metabólicas progresivas en el mundo asociado al riesgo de cambios metabólicos causados por la terapia antirretroviral se han considerado como factor exclusivo para la disfunción tiroidea, de igual forma la ausencia de terapia (Draenert, 2015). Por lo cual, se analizó la influencia de ciertos esquemas de terapia antirretroviral de gran actividad, tiempo de uso y etapa de infección en la función tiroidea, así mismo; se describieron los síntomas del paciente con disfunción tiroidea, por medio de la medición y análisis de hormonas tiroideas (TSH y T4 libre). Se determinó la presencia de hipotiroidismo subclínico la cual se relacionó con el tiempo de uso de antirretrovirales, y con el uso de profilaxis para infecciones oportunistas.

\section{Materiales y métodos}

Se realizó un estudio analítico transversal, evaluando a 218 pacientes con un muestreo probabilístico aleatorizado de pacientes según número correlacional, divididos en subgrupos según el tiempo de tratamiento antirretroviral de gran actividad (TARGA), atendidos en la Clínica de Atención Integral de Zacapa, cuya población reportada para el tiempo de la investigación era de 504 pacientes, se les realizó TSH y T4 libre mediante la extracción de una muestra de sangre y se interrogó acerca de síntomas relacionados con patología tiroidea, asociándolo con tiempo de la terapia, carga viral y CD4. Los datos fueron resumidos a través del cálculo de frecuencias y porcentajes en el caso de las variables cualitativas y medidas de tendencia central y de dispersión en el caso de variables cuantitativas.

\section{Resultados}

Con un muestreo probabilístico aleatorio de 218 pacientes estratificándolo en subgrupos en relación a los años de uso de terapia antirretroviral de gran actividad se describió la frecuencia con la que se presentó hipotiroidismo subclínico la cual fue de $8.3 \%$ e hipotiroidismo central en $3.2 \%$ de los pacientes. $\mathrm{Al}$ evaluar asociación entre disfunción tiroidea, se demostró una asociación significativa entre el tiempo de uso de antirretrovirales y la prevalencia de disfunción tiroidea $(\mathrm{p}<0.001)$, con una prevalencia de hipotiroidismo subclínico en una cuarta parte de los pacientes que han usado antirretrovirales por más de diez años (cuadro 1). 


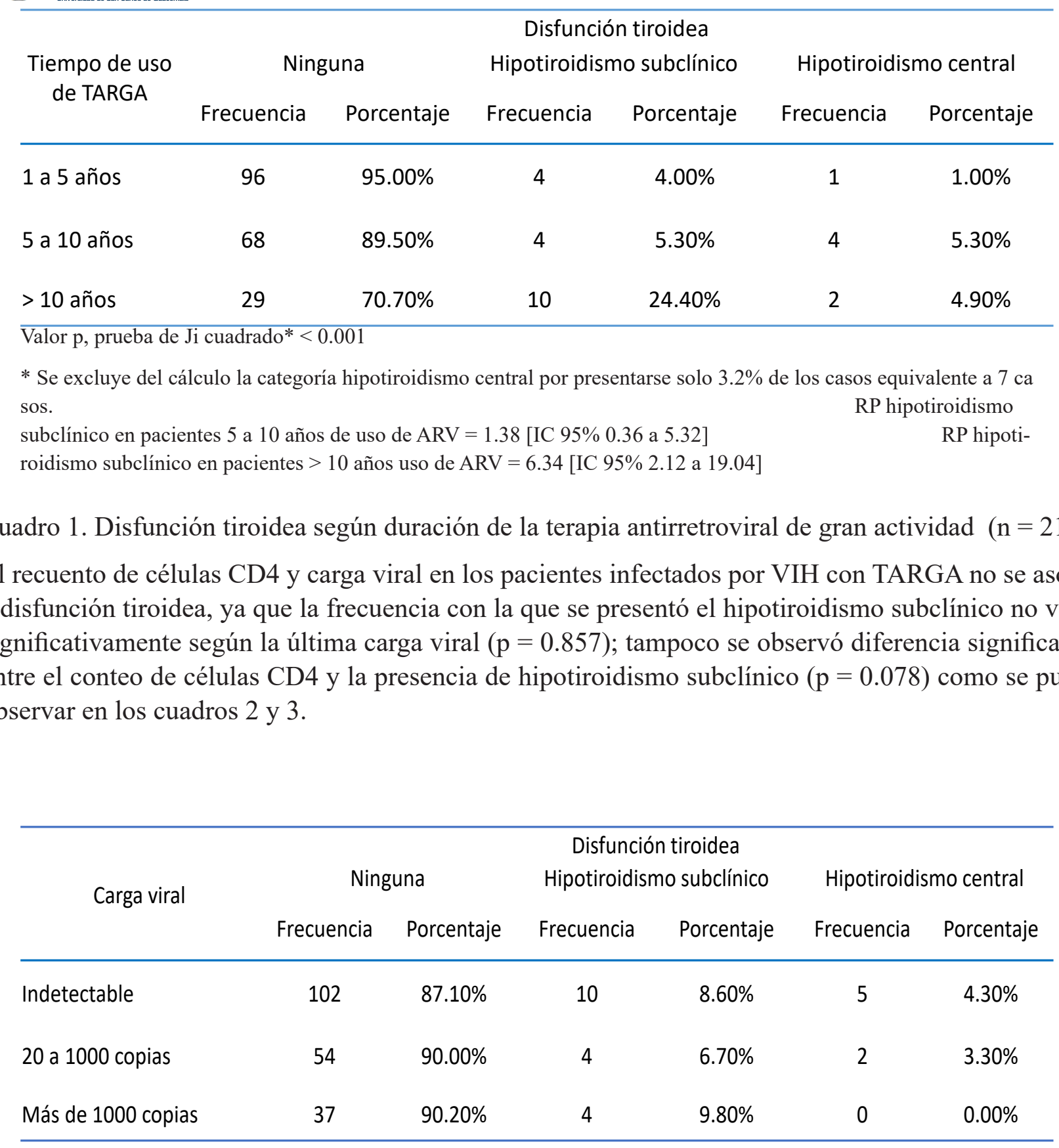

Valor $\mathrm{p}$, prueba de ji cuadrado $*=0.857$

* Se excluye del cálculo la categoría hipotiroidismo central por presentarse solo $3.2 \%$ de los casos equivalente a 7 casos.

Cuadro 2. Disfunción tiroidea según la última carga viral de los pacientes infectados por VIH $(\mathrm{n}=218)$. 


\begin{tabular}{ccccccccc}
\hline & \multicolumn{4}{c}{$\begin{array}{c}\text { Disfunción tiroidea } \\
\text { Ninguna }\end{array}$} & \multicolumn{3}{c}{ Hipotiroidismo subclínico } & \multicolumn{3}{c}{ Hipotiroidismo central } \\
\hline CD4+ & Q3 & Mediana & Q1 & Q3 & Mediana & Q1 & Q3 \\
Mediana & Q1 & Q3 & CD4+ \\
486 & 291 & 645 & 490 & 239 & 497 & 510 & 157 & 561 \\
\hline
\end{tabular}

Valor p, prueba de Mann-Whitney* $=0.078$

* Se excluye del cálculo la categoría hipotiroidismo central por presentarse solo $3.2 \%$ de los casos equivalente a 7 casos.

Cuadro 3. Relación del recuento de CD4 según el tipo de disfunción tiroidea $(\mathrm{n}=218)$.

Los esquemas antirretrovirales donde se presentó mayor porcentaje de casos de hipotiroidismo subclínico fueron TDF + FTC + NVP (18.2\%), el AZT + 3TC + EFV (15.0\%), TDF + FTC + EFV (8.1\%), se hizo una evaluación de la asociación con los medicamentos de forma individual pero no se encontró diferencias entre ellos. Hubo una diferencia estadísticamente significativa en la prevalencia de hipotiroidismo subclínico y la administración de profilaxis para infecciones oportunistas $(p=0.004)$ como se observa en el cuadro 4.

Se comparó la intensidad de los síntomas en los pacientes con disfunción tiroidea y los pacientes eutiroideos, los últimos prácticamente afirmaron no padecer de ninguno de los síntomas cuestionados. Sin embargo, se observó que había una mayor intensidad del insomnio y piel seca en pacientes con hipotiroidismo subclínico; y una mayor intensidad de debilidad y alteraciones menstruales en los pacientes con hipotiroidismo central.

\begin{tabular}{|c|c|c|c|c|c|c|c|}
\hline \multirow{3}{*}{\multicolumn{2}{|c|}{ Profilaxis }} & \multicolumn{6}{|c|}{ Disfunción tiroidea } \\
\hline & & \multicolumn{2}{|c|}{ Ninguna } & \multicolumn{2}{|c|}{ Hipotiroidismo subclínico } & \multicolumn{2}{|c|}{ Hipotiroidismo central } \\
\hline & & Frecuencia & Porcentaje & Frecuencia & Porcentaje & Frecuencia & Porcentaje \\
\hline \multirow{2}{*}{ Profilaxis } & No & 179 & $89.90 \%$ & 13 & $6.60 \%$ & 7 & $3.50 \%$ \\
\hline & Sí & 14 & $73.70 \%$ & 5 & $26.30 \%$ & 0 & $0.00 \%$ \\
\hline \multirow{7}{*}{ Tipo de profilaxis } & Ninguno & 179 & $89.90 \%$ & 13 & $6.60 \%$ & 7 & $3.50 \%$ \\
\hline & TMP-SMX & 4 & $57.10 \%$ & 3 & $42.90 \%$ & 0 & $0.00 \%$ \\
\hline & $\begin{array}{l}\text { TMP-SMX y } \\
\text { azitromicina }\end{array}$ & 2 & $66.70 \%$ & 1 & $33.30 \%$ & 0 & $0.00 \%$ \\
\hline & TMP-SMX y fluconazol & 3 & $100.00 \%$ & 0 & $0.00 \%$ & 0 & $0.00 \%$ \\
\hline & $\begin{array}{l}\text { TMP-SMX, azitromicina } \\
\text { e itraconazol }\end{array}$ & 1 & $100.00 \%$ & 0 & $0.00 \%$ & 0 & $0.00 \%$ \\
\hline & $\begin{array}{l}\text { TMP-SMX, azitromicina } \\
\text { y fluconazol }\end{array}$ & 3 & $75.00 \%$ & 1 & $25.00 \%$ & 0 & $0.00 \%$ \\
\hline & $\begin{array}{l}\text { TMP-SMX, } \\
\text { Azitromicina, fluconazol } \\
\text { e IRPE }\end{array}$ & 1 & $100.00 \%$ & 0 & $0.00 \%$ & 0 & $0.00 \%$ \\
\hline
\end{tabular}

Valor p, prueba de ji cuadrado $*=0.004$

* Se ex-

cluye del cálculo la categoría hipotiroidismo central por presentarse solo $3.2 \%$ de los casos equivalente a 7 casos. RP de hipotiroidismo subclínico en pacientes con profilaxis $=3.81$ [IC 95\% 1.55 a 9.36]RP de hipotiroidismo subclínico en pacientes con profilaxis $=3.81[$ IC 95\% 1.55 a 9.36]

Cuadro 4. Frecuencia de disfunción tiroidea según medicamentos profilácticos administrados para infecciones oportunistas $(n=218)$. 


\section{Discusión}

Se demostró que existe relación de disfunción tiroidea en pacientes infectados por el virus de la inmunodeficiencia humana con el tratamiento antirretroviral de gran actividad. La disfunción tiroidea más frecuente encontrada fue el hipotiroidismo subclínico lo cual es concordante con lo publicado hasta ahora. Las disfunciones tiroideas son más frecuentes a medida que aumenta tiempo de uso de antirretrovirales, lo cual se puede deber al compromiso inmunológico que presenta los pacientes con más de 10 años de infección por VIH y TARGA, pudiendo tener efectos sobre el sistema endocrino, o ser una complicación endocrinológica propia de la terapia. Si bien se ha estudiado que la disfunción tiroidea podría ser predictor de progresión a SIDA, la variable que podría reflejar mejor la progresión es el conteo de células CD4, pero en estos pacientes solo 29 de 218 tenían CD4 por debajo de 200 , valor que se considera de riesgo para la aparición de infecciones oportunistas. Los pacientes con profilaxis para oportunistas presentaron 3.8 veces la probabilidad de padecer hipotiroidismo subclínico en relación con los que no tenían profilaxis, lo cual podría deberse al efecto del fármaco en el sistema inmunitario.

Relacionando los datos estadísticos obtenidos con estudios internacionales, concuerda con los datos encontrados en Chile y China con Guatemala, en el cual indica una prevalencia de hipotiroidismo subclínico respectivamente $7.87 \%, 8.7 \%$ - 8.3\% (Ji, et al. 2016). Observando de la misma manera los datos del estudio realizado en Chile en donde se evalúan múltiples variables de principal importancia, como lo es la duración de la terapia antirretroviral, no fue significativo ( $p=0.392)$; en cambio, en Guatemala si fue significativo, principalmente en el grupo con exposición a la terapia por más de 10 años $(\mathrm{p}<0.001)$, tomando en cuenta que en este estudio los subgrupos se distribuyeron de una manera más específica (Baez, et al. 2016). Aunque no se logró demostrar una mayor prevalencia de disfunción tiroidea en este grupo de pacientes respecto a lo publicado en otras investigaciones, consideramos recomendable monitorizar la función tiroidea, ya que las manifestaciones clínicas de esta no son específicas y podrían deteriorar la calidad de vida y el pronóstico de los pacientes infectados por el VIH a largo plazo.

\section{Agradecimientos}

Al equipo médico y paramédico de la Clínica de Atención Integral de Zacapa, Guatemala, por la valiosa colaboración en el desarrollo de esta investigación, sin lo cual no habría sido posible. Nuestros agradecimientos al equipo del laboratorio clínico del Hospital Regional de Zacapa, por su cooperación en el procesamiento de las pruebas. A la Dra. Claudia Mazariegos, especialista en medicina interna y master en infectología de adultos, coordinadora de Clínica de Atención Integral de Zacapa, le agradecemos la asesoría prestada y revisión del manuscrito. A la Dra. Geraldina Utrilla, especialista en medicina interna y máster en endocrinología de adultos, por el interés y revisión del manuscrito.

\section{Referencias bibliográficas}

Báez, MS; Zapata S., López B. \& Wilson, G. (2016). Alteraciones tiroideas en pacientes infectados con el virus de inmunodeficiencia humana (en línea). Revista Médica de Chile 144(3):333-340. https:// doi.org/10.4067/S0034-98872016000300008 
Draenert, R. (2015) Pathogenesis of HIV-1 infection (en línea). In HIV 2015/16. Hoffmann, C; Rockstroh, JK (eds.). Hamburg, Alemania, Medizin Fokus Verlag. p. 22-750. Disponible en https:// hivbook.files.wordpress.com/2016/04/hiv-2015-16-complete.pdf

Ji, S., Jin, C., Höxtermann, S., Fuchs, W., Xie, T., Lu, X., Wu, H., Cheng, L., Skaletz-Rorowski, A., Brockmeyer, N \& Wu, N. (2016) Prevalence and influencing factors of thyroid dysfunction in HIV-infected patients (en línea). BioMed Research International 2016:1-3. https://doi.org/10.1155/2016/3874257

\section{Sobre la autora}

\section{Kristha García}

Médica y Cirujana egresada del Centro Universitario de Oriente CUNORI de la Universidad de San Carlos de Guatemala. Ha realizado investigaciones sobre la disfunción tiroidea en pacientes infectados por VIH con terapia antirretroviral.

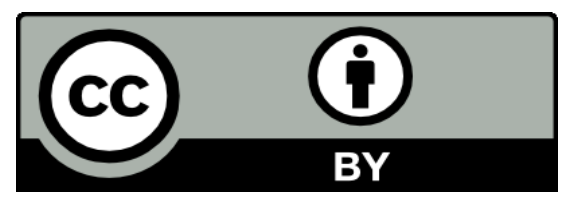

Este texto está protegido por una licencia CreativeCommons 4.0.

Usted es libre para compartir, copiar y redistribuir el material en cualquier medio o formato y adaptar el documento, remezclar, transformar y crear a partir del material para cualquier propósito, incluso comercialmente, siempre que cumpla la condición de atribución: usted debe reconocer el crédito de una obra de manera adecuada, proporcionar un enlace a la licencia, e indicar si se han realizado cambios. Puede hacerlo en cualquier forma razonable, pero no de forma tal que sugiera que tiene el apoyo del licenciante o lo recibe por el uso que hace. 